Artículo Original

Propuesta de mejora de rentabilidad mediante la optimización de procesos en el montaje de estaciones de radio base del tipo FAST SITE, llave en mano y colocalizado de una empresa del sector de telecomunicaciones

\title{
Proposal to improve profitability through the optimization of processes in the assembly of base radio stations of the FAST SITE type, turnkey and placed of a company in the telecommunications sector
}

\author{
Da Silva Zárate, J . ${ }^{1}$, Narváez Benítez, F. C. ${ }^{1}$ \\ ${ }^{1}$ Universidad del Cono Sur de las Américas. Carrera de Ingeniería Industrial, \\ Asunción, Paraguay
}

\section{RESUMEN}

El propósito de este trabajo de investigación fue dar una propuesta de mejora de rentabilidad, teniendo como premisa la optimización de procesos en el montaje, específicamente de tres tipos de estaciones de radio base: Fast site, llave en mano y colocalizado de una empresa del rubro de las telecomunicaciones, y de ese modo evitar multas por retraso de obras, derivados de la falta de disponibilidad de materiales y considerando que el sistema de trabajo actual no permite cuantificar el costo del montaje de las estaciones. El desarrollo de la investigación de alcance descriptivo consistió en la recolección de información correspondientes a cuadernos técnicos, investigaciones, el análisis de la industria partiendo del relevamiento de datos, así como también entrevistas al personal de Ingeniería de la industria. El estudio determinó la ausencia de un inventario de materiales mínimo para asegurar la continuidad de la ejecución del montaje de las estaciones de radio base, esto debido a que no disponían de ningún control sobre los materiales a utilizar, para ello se concluyó que para el mejoramiento de la rentabilidad en el montaje se debe establecer un método de trabajo con permita un flujo continuo, con un inventario mínimo de materiales en depósito, el diseño de un listado estándar de materiales necesarios por cada tipo de estación y un método de medición y control que permita generar información que sirva como retroalimentación a todo el conjunto de procesos que forman parte del montaje de una estación de radio base de cada tipo citado anteriormente.

Palabras clave: rentabilidad, optimización, estación de radio base, procesos, montaje.

\begin{abstract}
The purpose of this research work was to give a proposal for improving profitability, having as a premise the optimization of processes in the assembly, specifically of three types of radio base stations: Fast site, turnkey and colocalized of a company in the telecommunications area, and thus avoid fines for delay of works, derived from the lack of availability of materials and
\end{abstract}

*Autor correspondiente: Camila Narváez Benítez. Universidad del Cono Sur de las Américas. Carrera de Ingeniería Industrial

Correo electrónico: camilanarvaez@ieee.org

Fecha de recepción: junio 2020 Fecha de aceptación: agosto 2020

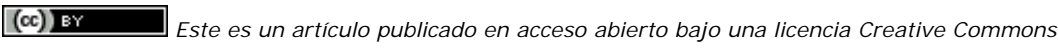


considering that the current system of work does not allow quantifying the cost of assembly of the stations. The development of the research of descriptive scope consisted of the collection of information corresponding to técnica notebooks, investigations, the analysis of the industry starting from the data collection, as well as interviews to the engineering personnel of the industry. The study determined the absence of a minimum inventory of materials to ensure the continuity of the execution of the assembly of the radio base stations, this due to the fact that they did not have any control over the materials to be used, for that reason it was concluded that for the improvement of the profitability in the assembly a work method with a continuous flow must be established, with a minimum inventory of materials in deposit, the design of a standard list of materials required by each type of station and a method of measurement and control that allows the generation of information that serves as feedback to the entire set of processes that are part of the assembly of a radio base station of each type mentioned above.

Keywords: profitability, optimization, radio base stations, processes, assembly.

\section{NTRODUCCI ÓN}

Para el desarrollo del Paraguay es sumamente relevante el despliegue de las redes de telecomunicaciones que permitan brindar mejores servicios a los consumidores. El Servicio de Telefonía Móvil / Banda Ancha Móvil requiere de la implementación de Estaciones Radio Base eficientes y seguras, con la instalación de antenas colocadas en torres. El servicio de telecomunicaciones básico para la adecuada llegada de la sociedad de la información y la mejora de la calidad de vida de los ciudadanos, (CONATEL, 2016; Cinco Días, 2017).

Teniendo en cuenta lo mencionado anteriormente, el presente trabajo considera como premisa fundamental el continuo y adecuado despliegue de infraestructuras de telecomunicación, por lo que el montaje de las mismas exige la aplicación de métodos de trabajo que permitan un flujo de trabajo eficiente y efectivo donde se cumplan los plazos establecidos por la empresa contratante y consiga que la empresa contratista encargada no posea pérdidas económicas ajenas a las proyectadas en dicho proyecto (Comisión Europea, 2014; Del Río Ruíz, 2018; Inzaurralde et al, 2014).

Actualmente no existe en nuestro país una norma que regule el proceso de montaje de una estación de radio base, por consiguiente, tampoco se cuenta con parámetros que permitan tener indicadores de eficiencia, planes de monitoreo, ni se cuenta con un manual de implementación de un método de trabajo adecuado para los mismos, donde se puedan contemplar los costos de materiales y mano de obra utilizados para el montaje de los distintos tipos de estaciones de radio base.

Por lo expuesto se realizó este estudio con el objetivo de proponer una mejora de rentabilidad mediante la optimización de procesos para el montaje de estaciones de radio base del tipo Fast site, llave en mano y colocalizado de una empresa del sector de telecomunicaciones.

El estudio se efectuó con una proyección de 5 años, dicho trabajo de investigación tuvo su inicio en enero del año 2019, concluyendo finalmente en agosto del año 2019. Para el trabajo de campo se realizó una serie de evaluaciones de la industria a ser analizada, teniendo como premisa los antecedentes de problemas de multas por retraso de obras, derivados de la falta de disponibilidad de materiales y la falta de conocimiento de los costos 
asignados a cada obra que indicaban que presumiblemente eran debido a la falta de optimización de procesos, mediante un método de trabajo adecuado, dicho análisis inició el 03 de junio del 2019 y concluyó el 28 de junio del 2019, siendo como objeto de estudio los tres tipos de estaciones de radio base, específicamente del tipo Llave en mano ubicada en la ciudad de Guarambaré, del tipo Fast Site ubicada en la ciudad de Capiatá y del tipo colocalizado ubicada en San Lorenzo, montados por una industria ubicada en la ciudad de Fernando de la Mora, perteneciente al rubro metalúrgico.

\section{MATERI ALES Y MÉTODOS}

El análisis de la situación actual de la industria del presente caso de estudio tiene como finalidad evitar las penalizaciones económicas y conocer la cantidad de materiales y los respectivos costos reales del montaje de estaciones de radio base, considerando el promedio de pedidos anuales de los mismos, por lo tanto, el alcance de la investigación es del tipo descriptivo debido a que con los datos recolectados y las informaciones obtenidas, primero nos condujeron a establecer las pérdidas económicas, identificar las falencias en su método de trabajo actual, por consiguiente con el análisis de los mismos se obtuvo una perspectiva más amplia de la situación de la industria y nos llevaron a establecer la solución más factible para el caso del estudio, (Vivanco Vergara, 2017; Pérez Gutiérrez et al, 2014).

La muestra objeto de estudio son los tres tipos de estaciones de radio base que son; los del tipo fast site, llave en mano y colocalizado cuya ejecución o montaje son realizados por una industria metalúrgica ubicada en la ciudad de Fernando de la Mora. En primer lugar, se realizó una recopilación y verificación de materiales bibliográficos, datos publicados en páginas web, artículos técnicos, revistas científicas, informes y datos provenientes de entrevistas realizados al personal de la industria de los departamentos de ingeniería y logística, (León, 2015; Ramírez, 2017).

El trabajo abarcó cuatro etapas:

Primera etapa: Recolección de datos del flujo y método de trabajo actual de la empresa, para poder realizar una optimización del sistema de trabajo existente fue necesario la recolección y el estudio de todo el proceso, teniendo que recurrir a entrevista del personal del departamento de ingeniería y logística.

Segunda etapa: Estudio del método de trabajo actual, se realizó un estudio exhaustivo del flujo de trabajo actual de la industria, logrando asi detectar todas las falencias existentes en el sistema, realizando observaciones y dejando un registro correspondiente de los mismos.

Tercera etapa: en base al estudio realizado en la etapa dos se elaboró la propuesta del nuevo método de trabajo, la misma consiste en estandarizar el proceso de montaje de las estaciones de radio base. También se diseñaron indicadores y un plan de monitoreo para lograr así dar una retroalimentación de todas las actividades, materiales utilizados, tiempos y costos que conlleva el montaje de una estación.

Cuarta etapa: Evaluación de la viabilidad de la propuesta de aplicación mediante herramientas que cuantifiquen el costo/beneficio.

Para cuantificar y registrar el sistema de trabajo actual recurrimos al uso de técnicas de medición y verificación del método de trabajo actual, a fin de realizar un análisis y verificación en la calidad del servicio brindado por la 
industria. Con los resultados obtenidos en la medición se comparó los datos obtenidos con los valores que otras industrias están logrando y también la calidad de los productos y servicios que la directiva espera con la que se obtiene, por lo tanto, resultó necesario la revisión de normas internacionales sobre calidad. El diseño del método de trabajo adecuado, así como el dimensionamiento de los paquetes estándar de materiales requirió operaciones matemáticas que permitan obtener las características y especificaciones técnicas del mismo.

\section{RESULTADOS}

Análisis de la situación actual de la empresa analizada con respecto al montaje de estaciones de radio base

La empresa analizada posee una cantidad mínima de montajes de estaciones de radio base asignadas anualmente por los clientes, dichas obras en su mayoría poseen especificaciones técnicas establecidas.

Tabla 1: Estaciones de Radio Base montadas en los últimos 5 años.

\begin{tabular}{|c|c|c|c|c|c|}
\hline $\begin{array}{l}\mathbf{A} \\
\tilde{\mathbf{n}} \\
\mathbf{0}\end{array}$ & Tipo & Torre & Cantidad & Total & $\begin{array}{c}\text { Porcentaje } \\
\text { de } \\
\text { Producción }\end{array}$ \\
\hline \multirow{3}{*}{$\begin{array}{l}2 \\
0 \\
1 \\
5\end{array}$} & Fast Site & Monopolo & 28 & \multirow{3}{*}{85} & $40 \%$ \\
\hline & $\begin{array}{l}\text { Llave en } \\
\text { Mano }\end{array}$ & TAS/ MASTI L & 27 & & \\
\hline & Colocalizado & $\mathbf{N} / \mathbf{A}$ & 30 & & \\
\hline \multirow{3}{*}{$\begin{array}{l}2 \\
0 \\
1 \\
6\end{array}$} & Fast Site & Monopolo & 13 & \multirow{3}{*}{51} & \multirow{3}{*}{$24 \%$} \\
\hline & Llave en Mano & TAS/MASTIL & 11 & & \\
\hline & Colocalizado & $\mathrm{N} / \mathrm{A}$ & 27 & & \\
\hline \multirow{3}{*}{$\begin{array}{l}2 \\
0 \\
1 \\
7\end{array}$} & Fast Site & Monopolo & 10 & \multirow{3}{*}{17} & \multirow{3}{*}{$8 \%$} \\
\hline & $\begin{array}{l}\text { Llave en } \\
\text { Mano }\end{array}$ & TAS/ MASTI L & 4 & & \\
\hline & Colocalizado & $\mathbf{N} / \mathbf{A}$ & 3 & & \\
\hline \multirow{3}{*}{$\begin{array}{l}2 \\
0 \\
1 \\
8\end{array}$} & Fast Site & Monopolo & 3 & \multirow{3}{*}{29} & \multirow{3}{*}{$14 \%$} \\
\hline & Llave en Mano & TAS/MASTIL & 2 & & \\
\hline & Colocalizado & N/A & 24 & & \\
\hline \multirow{3}{*}{$\begin{array}{l}2 \\
0 \\
1 \\
9\end{array}$} & Fast Site & Monopolo & 4 & \multirow{3}{*}{30} & \multirow{3}{*}{$14 \%$} \\
\hline & Llave en Mano & TAS/MASTIL & 9 & & \\
\hline & Colocalizado & N/A & 17 & & \\
\hline
\end{tabular}

Fuente: Elaboración propia.

Haciendo un análisis de los valores mencionados anteriormente en los últimos 5 años, se puede observar en la siguiente figura 1 que, la mayor cantidad de sitios realizados fue en el año 2015, donde se totalizaron de 85 sitios, es decir con $40 \%$ de producción de la producción total, sin embargo, en el año 2017 se 
obtuvo la menor cantidad de sitios realizados totalizando 17 sitios, con solamente el $8 \%$ de la producción total.

Posteriormente en los años 2018 y 2019, la producción mantuvo la disminución de asignación de obras de estaciones de radio base, contabilizando 29 y 30 sitios por año respectivamente.

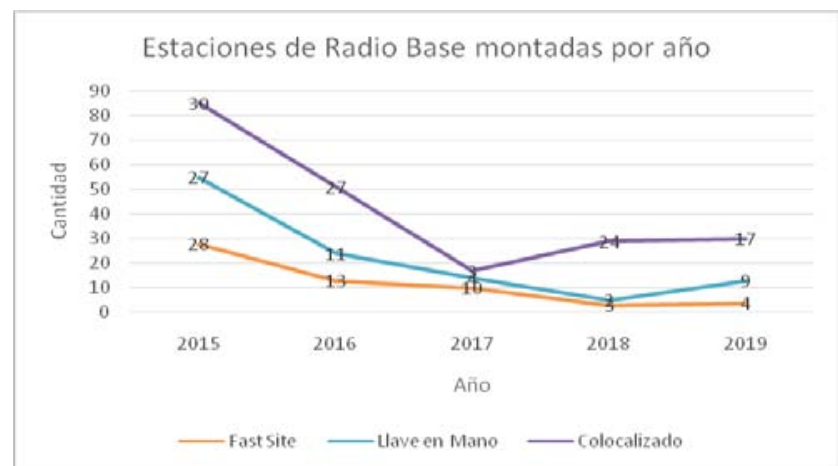

Figura 1: Estaciones de Radio Base montadas en los últimos 5 años. Fuente: Elaboración propia.

Teniendo en cuenta el tipo de estación de radio base se puede observar, en las figuras 2, 3 y 4 se observan de manera desglosada la cantidad de estaciones de radio base.

\section{Estaciones de radio base del tipo Fast Site montadas por año}

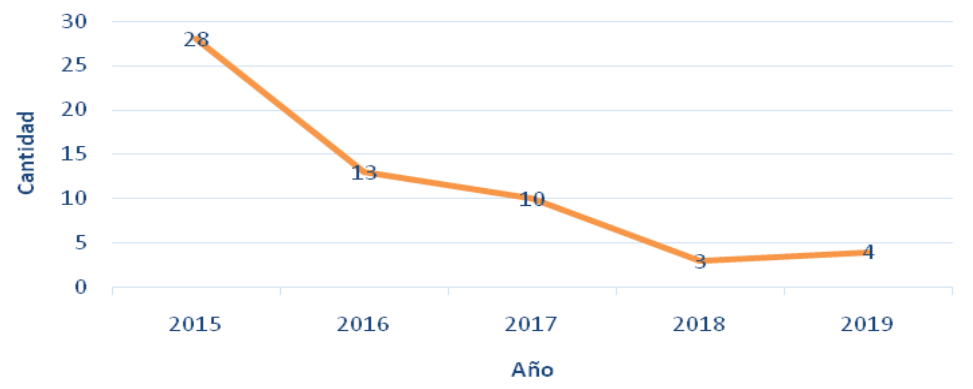

Figura 2: Estaciones de radio base del tipo Fast Site.

Fuente: Elaboración propia. 


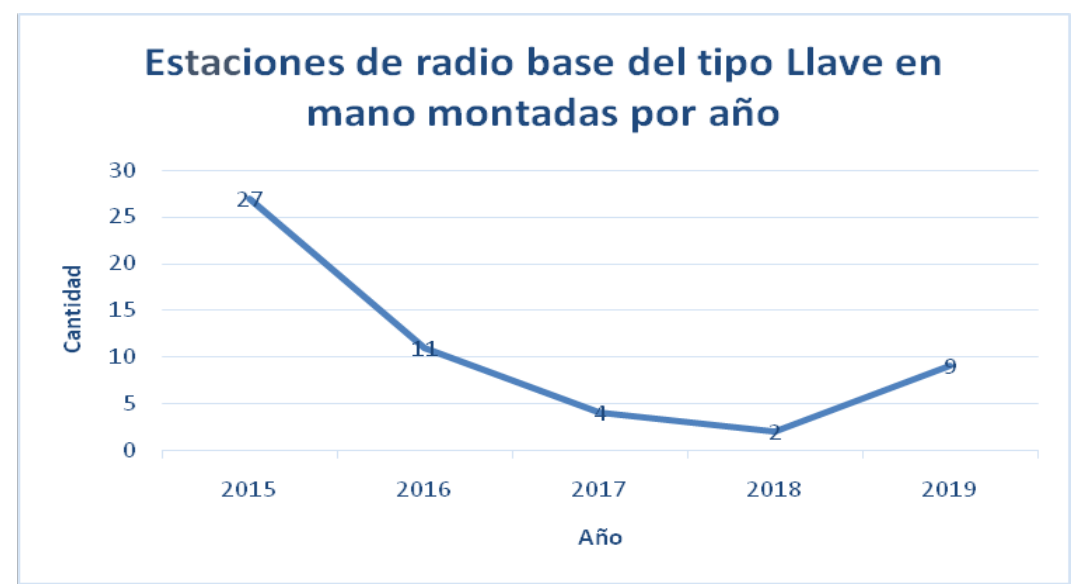

Figura 3: Estaciones de radio base del tipo Llave en mano. Fuente: Elaboración propia.

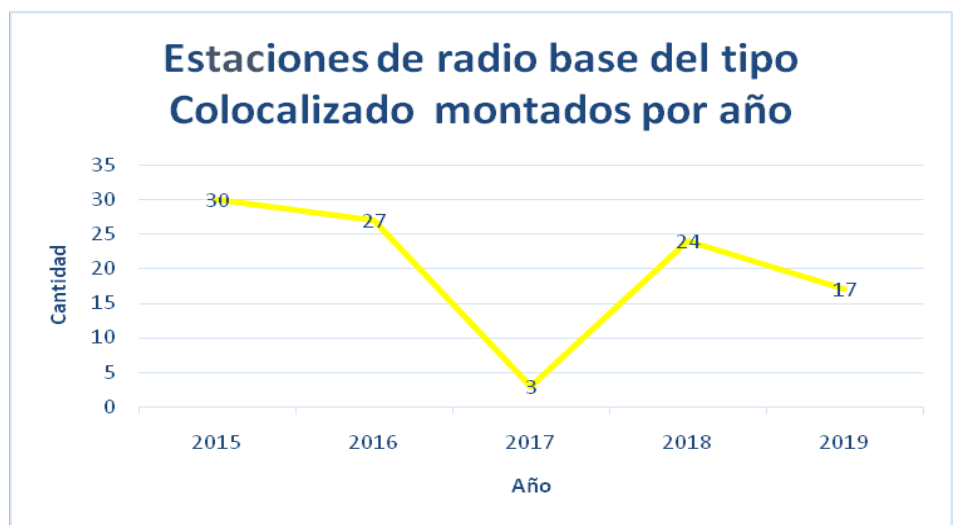

Figura 4: Estaciones de radio base del tipo Colocalizado.

Fuente: Elaboración propia.

A continuación, se muestra el cálculo del promedio de la cantidad de sitios de cada tipo de estaciones de radio base y los resultados se detallan en la tabla 2:

Fast Site

$P=\frac{28+13+10+3+4}{5}=11.6 \cong 12$

Llave en mano

$P=\frac{27+11+4+2+9}{5}=10.6 \cong 11$

Colocalizado 


$$
P=\frac{30+27+3+24+17}{5}=20.2 \cong 20
$$

\begin{tabular}{|l|l|l|}
\hline TI PO & MORRE & CANTI DAD \\
\hline Fast Site & Monopolo & 12 \\
\hline Llave en Mano & TAS/MASTIL & 11 \\
\hline Colocalizado & N/A & 20 \\
\hline
\end{tabular}

Tabla 2: Promedio de estaciones de radio base realizadas por año. Fuente: Elaboración propia.

En la Tabla 2 se demuestra que se han montado anualmente más estaciones de radio base del tipo colocalizado que las del tipo Fast Site y Llave en mano.

\section{Problemas encontrados en el montaje de estaciones de radio base en los últimos 5 años}

Teniendo en cuenta la entrevista realizada al Gerente de Operaciones, líder del Departamento de Ingeniería y el Jefe de Logística de la industria analizada, a continuación, se enumeran los problemas principales:

\section{Falta de organigrama jerárquico y definición de funciones}

La falta de un organigrama oficial en la empresa hace que se trabaje de manera desordenada y sin coordinación, muchas veces las decisiones dependen de un único superior. En ocasiones los trabajadores hacen tareas que no les corresponde, ya que no están bien definidas las funciones.

\section{Tercerización del área civil y eléctrica}

El mayor costo se encuentra en la tercerización de la mano de obra civil y eléctrica, ya que solamente se cuenta con un solo proveedor en cada área desde los últimos 5 años, y no es posible conocer de manera desglosada el precio exacto de cada material necesario.

3. Exceso de reuniones improductivas

Se tienen como mínimo 5 reuniones al día con el gerente general, en caso del gerente de operaciones 8 a 10 reuniones a lo largo del día teniendo un promedio de tiempo de 30 minutos. Citando al encargado de logística "4 de las 5 reuniones que tengo en el día no son para tomar decisiones, lo que resulta en pérdida de tiempo y ritmo de trabajo constante que debo de tener".

4. Falta de control en los procesos

Los procesos involucrados para el montaje de las estaciones de radio base carecen de control, dificultando una retroalimentación para el mejoramiento de procesos, por consiguiente, en ciertos procesos críticos, existe pérdida de tiempo, resultando en pérdidas monetarias.

\section{Falta de control de materiales y herramientas}

Los materiales y herramientas son entregados a veces por el encargado de logística y en otras ocasiones son retiradas directamente del depósito por los montadores o personal del departamento de herrería sin ser registrado su retiro o uso. Como 
resultado se tiene un registro no fiable del cual se pueda elaborar un inventario o dar seguimientos a las herramientas y materiales.

\section{Falta de control de uso de materiales}

Los materiales eléctricos, civiles y metálicos son hechos en base al pedido de materiales realizado por el departamento de ingeniería.

No se dispone un registro de los materiales que fueron utilizados para el montaje de las ERB, por lo tanto, no se puede saber la cantidad de materiales utilizados con exactitud en el montaje de los sitios.

\section{Vehículos insuficientes}

Todos los vehículos son ocupados para el montaje de los sitios, pero en ocasiones se deja de lado las gestiones administrativas (pago a proveedores, autorizaciones, retiro/entrega de facturas, etc.) y las entregas y gestiones de documentos (permisos municipales, reuniones con clientes, etc.).

\section{Falta de capacitación del personal}

El personal de las áreas involucradas (civil, eléctrico, herrería etc.), en el montaje de estaciones de radio base, comete errores en sus rubros por desconocimiento de tareas o técnicas para realizar algunos procesos.

Los problemas mencionados anteriormente generan multas por parte del cliente debido a los retrasos en el montaje, ya que se encuentran establecidos los porcentajes anuales a ser cobrados en concepto de multa, correspondiente a $9 \%$ por cada estación de radio base, traducidos en grandes pérdidas económicas para la industria analizada.

\section{Método de trabajo propuesto \\ Relevamiento de datos}

Se realizó la medición y relevamiento de manera a establecer primeramente los materiales y segundo la mano de obra necesarios para el montaje de cada tipo de estación de radio base. Ver las figuras 5, 6, 7 y 8 que se me muestran a continuación:

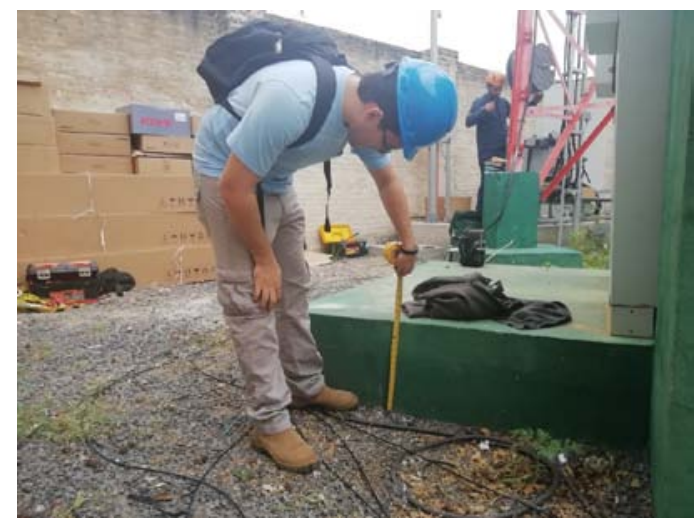

Figura 5: Medición de la platea de la estación. 
Fuente: Elaboración propia.

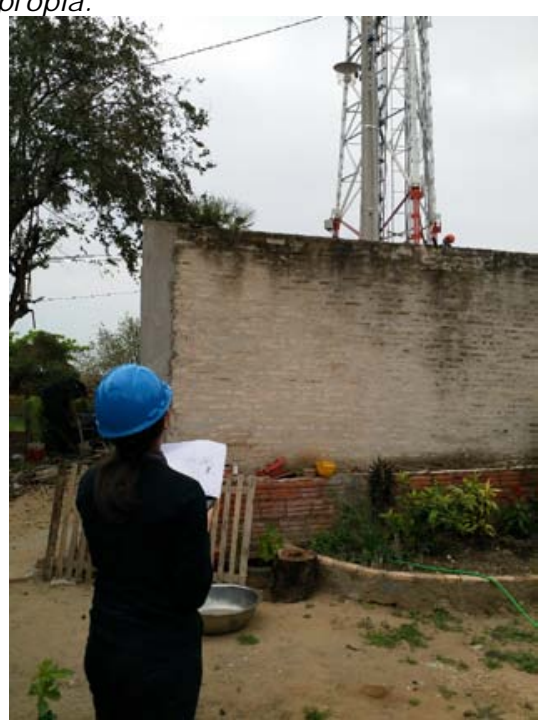

Figura 6: Registro de las dimensiones de la torre. Fuente: Elaboración propia.

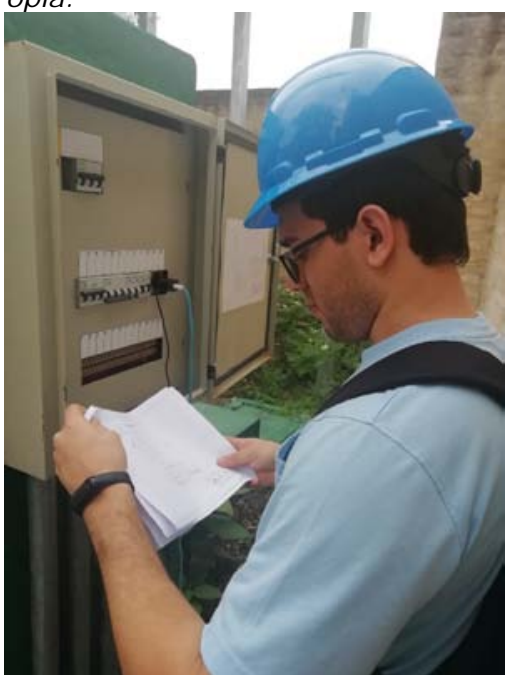

Figura 7: Revisión de los planos eléctricos.

Fuente: Elaboración propia. 


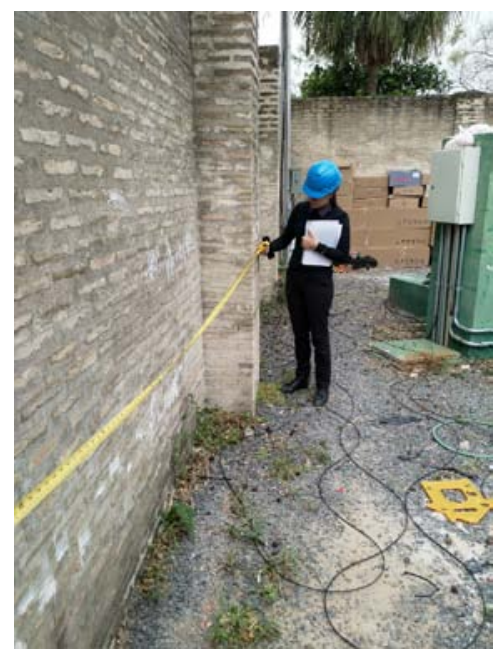

Figura 8: Medición de las dimensiones del terreno.

Fuente: Elaboración propia.

\section{Manual de Procedimientos propuesto}

Consideramos que un manual de procedimiento explicaría de manera detallada los procedimientos del montaje, asi también a través del mismo se podría evitar grandes errores que se suelen cometer dentro de las áreas funcionales de la empresa analizada. Por lo tanto, se procedió a plasmar la propuesta de solución en el siguiente el manual propuesto, cuyo contenido se expone a continuación:

\section{Capacitación en el uso del manual propuesto}

Teniendo como prioridad la capacitación de los trabajadores en el uso del manual, se ofrecerá la oportunidad ideal para que continúen ampliando sus conocimientos en el rubro, para trabajar de manera eficiente y efectiva en todas las áreas involucradas. Una planificación adecuada del componente humano en una organización requiere de una política de capacitación permanente, se sugiere que, para la etapa inicial de la implementación del método de trabajo, se realicen una capacitación al mes, durante 6 meses. Posteriormente se evaluará la frecuencia en las capacitaciones, de acuerdo con la recepción de la información por parte de los trabajadores

Cada líder de cada departamento involucrado en el montaje de estaciones de radio base tendrá que realizar informes de avance en cada capacitación, especificando la fecha, horario y personas capacitadas, de manera a llevar un registro, controlando constantemente que cada trabajador de cada área cumpla con las tareas establecidas durante la capacitación. Dichos informes tendrán que ser remitidos al Departamento de Operaciones.

El Departamento de Operaciones es el encargado de verificar el cumplimiento de lo establecido en el manual propuesto, ya que el Gerente de Operaciones tendrá reuniones mensuales con el Gerente General para evaluar la situación actual de la empresa, estableciendo mejoras y estrategias, facilitando 
de esta manera la toma de decisiones para lograr la correcta implementación del método propuesto en el presente manual.

\section{Análisis económico de la propuesta presentada}

Teniendo como foco principal las penalidades por retraso en la obra, se encuentra contemplado la demora en el cumplimiento de las obligaciones previas a la recepción definitiva de la obra, la multa a aplicar será del $9 \%$ del monto total de la orden del trabajo en concepto del montaje de estaciones de radio base ya sea del tipo Fast Site, llave en mano y colocalizado por cada día de atraso sin justificación valedera a juicio del cliente, a partir de la fecha establecida.

Cabe destacar que el porcentaje de multa es aplicada hasta máximo 5 dias de retraso de obra con relación al cronograma establecido por la empresa, en caso de ser superada esta limitante, la empresa es sometida a una sanción que consiste en la suspensión de sus servicios como proveedor.

\section{Método actual}

Según la información brindada por la empresa analizada la cantidad de sitios multados reportados en los últimos 5 años se especifican en la siguiente Tabla 3.

Tabla 3: Cantidad de sitios multados utilizando el método actual.

\begin{tabular}{|c|c|c|c|c|c|c|c|}
\hline AÑO & TIPO DE SITIO & $\begin{array}{c}\mathrm{N}^{\circ} \mathrm{DE} \\
\text { MULTAS }\end{array}$ & COSTO SITIO & $\begin{array}{c}\text { MULTA } \\
\%\end{array}$ & MULTA GS & TOTAL MULTA & TOTAL ANUAL \\
\hline \multirow{3}{*}{2015} & FAST SITE & 5 & 34.000 .000 & $9 \%$ & 3.060 .000 & 15.300 .000 & \multirow{3}{*}{137.376 .000} \\
\hline & LLAVE EN MANO & 10 & 114.500 .000 & $9 \%$ & 10.305 .000 & 103.050 .000 & \\
\hline & \begin{tabular}{|l} 
COLOCALIZADO \\
\end{tabular} & 7 & 30.200 .000 & $9 \%$ & 2.718 .000 & 19.026 .000 & \\
\hline \multirow{3}{*}{2016} & FAST SITE & 6 & 34.000 .000 & $9 \%$ & 3.060 .000 & 18.360 .000 & \multirow{3}{*}{76.455 .000} \\
\hline & LLAVE EN MANO & 3 & 114.500 .000 & $9 \%$ & 10.305 .000 & 30.915 .000 & \\
\hline & COLOCALIZADO & 10 & 30.200 .000 & $9 \%$ & 2.718 .000 & 27.180 .000 & \\
\hline \multirow{3}{*}{2017} & FAST SITE & 3 & 34.000 .000 & $9 \%$ & 3.060 .000 & 9.180 .000 & \multirow{3}{*}{37.944 .000} \\
\hline & LLAVE EN MANO & 2 & 114.500 .000 & $9 \%$ & 10.305 .000 & 20.610 .000 & \\
\hline & COLOCALIZADO & 3 & 30.200 .000 & $9 \%$ & 2.718 .000 & 8.154 .000 & \\
\hline \multirow{3}{*}{2018} & FAST SITE & 3 & 34.000 .000 & $9 \%$ & 3.060 .000 & 9.180 .000 & \multirow{3}{*}{43.947 .000} \\
\hline & LLAVE EN MANO & 1 & 114.500 .000 & $9 \%$ & 10.305 .000 & 10.305 .000 & \\
\hline & COLOCALIZADO & 9 & 30.200 .000 & $9 \%$ & 2.718 .000 & 24.462 .000 & \\
\hline \multirow{3}{*}{2019} & FAST SITE & 2 & 34.000 .000 & $9 \%$ & 3.060 .000 & 6.120 .000 & \multirow{3}{*}{82.674 .000} \\
\hline & LLAVE EN MANO & 4 & 114.500 .000 & $9 \%$ & 10.305 .000 & 41.220 .000 & \\
\hline & COLOCALIZADO & 13 & 30.200 .000 & $9 \%$ & 2.718 .000 & 35.334 .000 & \\
\hline \multicolumn{2}{|r|}{ TOTAL 5 AÑOS } & 81 & & & & & 378.396.000 \\
\hline
\end{tabular}

Fuente: Proveído por la empresa analizada.

A continuación, se muestra el cálculo del promedio de la cantidad de sitios multados de cada tipo de estaciones de radio base:

Fast Site

$$
P=\frac{5+6+3+3+2}{5}=3.80 \cong 4
$$

Llave en mano

$$
P=\frac{10+3+2+1+4}{5}=4
$$

Colocalizado

$$
P=\frac{7+10+3+9+12}{5}=8,40 \cong 8
$$


En la tabla4, se observa el promedio de sitios con multa con sus respectivos porcentajes y asi también los montos promedio de multa incurridos anualmente, aplicando el método actual:

Tabla 4: Promedio de sitios con multa (actual).

\begin{tabular}{|c|c|c|c|c|}
\hline TIPO DE SITIO & $\begin{array}{l}\text { № DE MULTAS } \\
\text { PROMEDIO }\end{array}$ & $\begin{array}{l}\% \text { SITIO CON } \\
\text { MULTA }\end{array}$ & $\begin{array}{l}\% \text { SITIO SIN } \\
\text { MULTA }\end{array}$ & $\begin{array}{l}\text { COSTO ANUAL } \\
\text { DE LA MULTA }\end{array}$ \\
\hline FAST SITE & 4 & $2,85 \%$ & $97,15 \%$ & 11.628 .000 \\
\hline $\begin{array}{ll}\text { LLAVE } & \text { EN } \\
\text { MANO } & \end{array}$ & 4 & $3,27 \%$ & $96,73 \%$ & 41.220 .000 \\
\hline COLOCALIZADO & 8 & $3,78 \%$ & $96.22 \%$ & 22.831 .200 \\
\hline
\end{tabular}

Fuente: Elaboración propia.

Lo mencionado anteriormente se plasman en las siguientes Figuras 9 y 10:

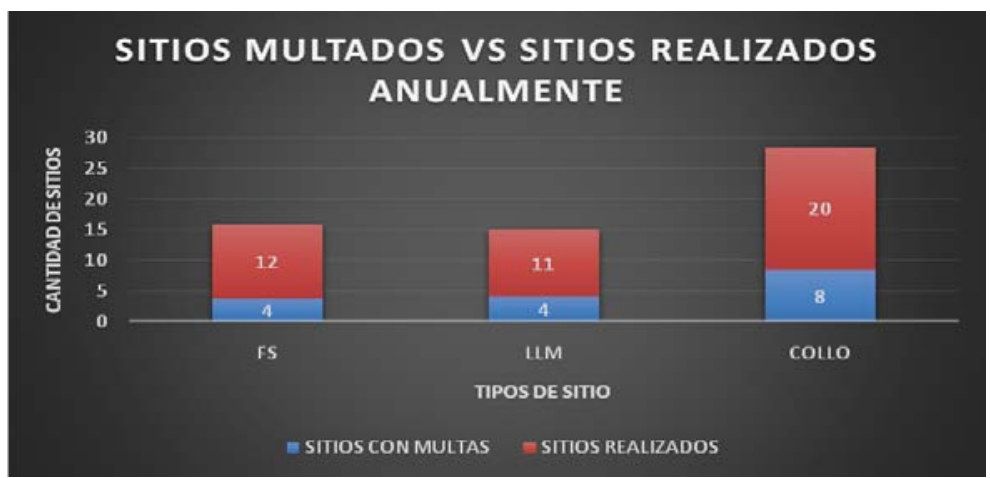

Figura 9: Sitios multados vs sitios realizados anualmente.

Fuente: Elaboración propia. 


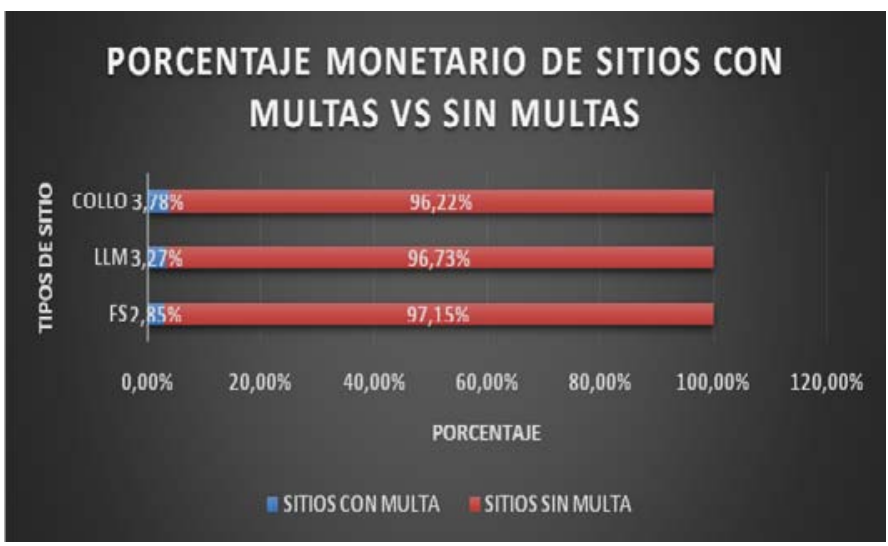

Figura 10: Porcentaje de sitios con multa.

Fuente: Elaboración propia.

\section{Método propuesto}

Se pretende disminuir las multas en un $50 \%$ aplicando el método propuesto. En la tabla5, se observa el promedio de sitios con multa con sus respectivos porcentajes y asi también los montos promedio en de multa incurridos anualmente, aplicando el método actual:

\begin{tabular}{|l|l|l|l|l|}
\hline TIPO DE SITIO & $\begin{array}{l}\text { No } \\
\text { MULTAS }\end{array}$ & $\begin{array}{l}\text { DE SITIO } \\
\text { CON } \\
\text { MULTA }\end{array}$ & $\begin{array}{l}\text { S SITIO } \\
\text { MULTA }\end{array}$ & $\begin{array}{l}\text { MONTO } \\
\text { ANUAL DE LA } \\
\text { MULTA }\end{array}$ \\
\hline FAST SITE & 2 & $1,50 \%$ & $98,00 \%$ & 3.754 .298 \\
\hline LLAVE EN MANO & 2 & $1,64 \%$ & $98,00 \%$ & 16.747 .649 \\
\hline COLOCALIZADO & 4 & $1,80 \%$ & $99,00 \%$ & 8.613 .464 \\
\hline
\end{tabular}

Tabla5: Promedio de sitios con multa (propuesto).

Fuente: Elaboración propia.

Lo mencionado anteriormente se plasman en las siguientes figuras 11 y 12 : 


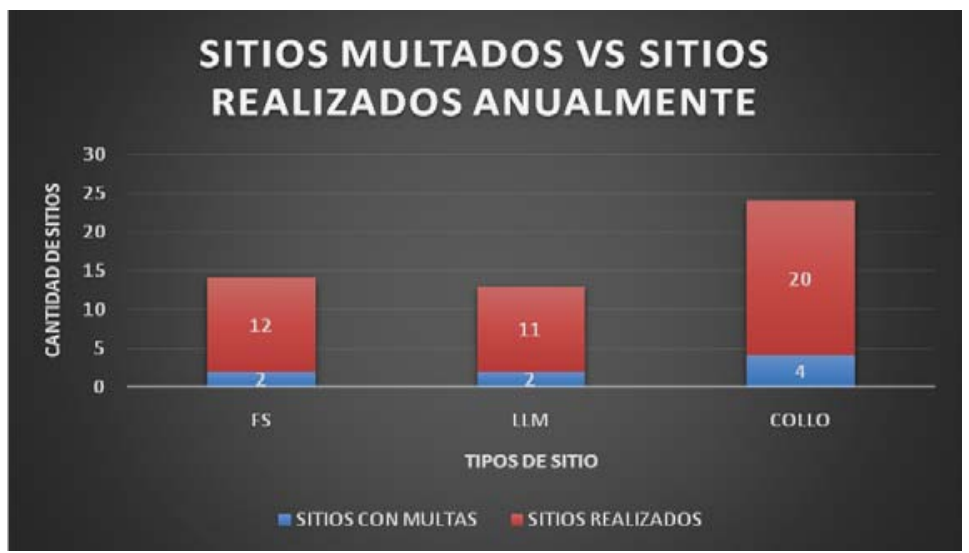

Figura 11: Sitios multados vs sitios realizados anualmente. Fuente: Elaboración propia.

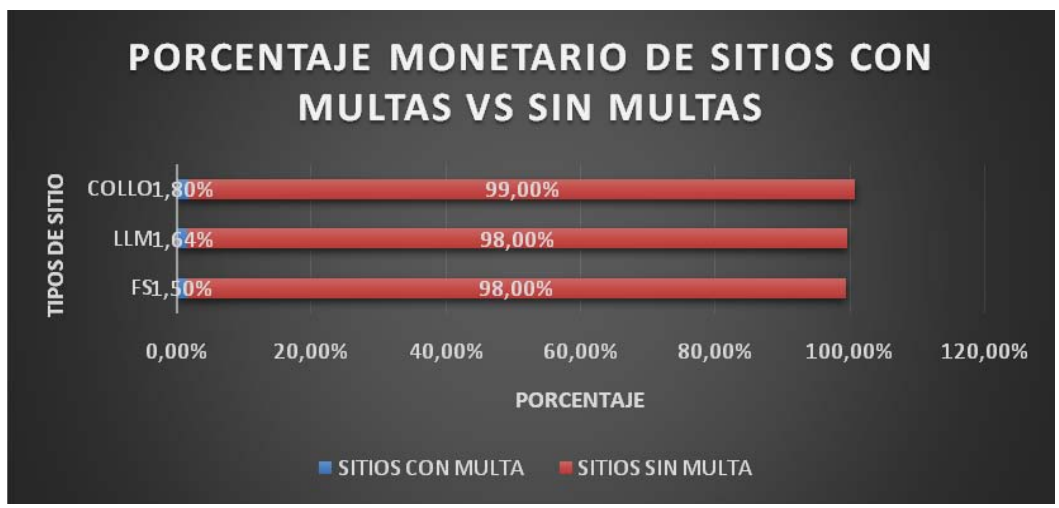

Figura 12: Porcentaje de sitios con multa.

Fuente: Elaboración propia.

\section{Análisis comparativo entre el método actual y el método propuesto}

Se han demostrado las consecuencias técnico-económica negativas que redujeron la eficiencia de en el montaje de estaciones de radio base en la industria. Desde el punto de vista económico se puede concluir que si se llegara a implementar el método de trabajo propuesto sería viable. (Ver Tabla 6).

Tabla 6: Análisis de costos del montaje con el método actual vs método propuesto.

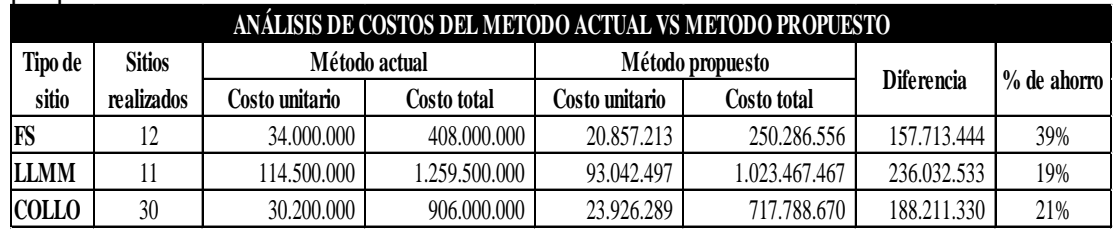


Fuente: Elaboración propia.

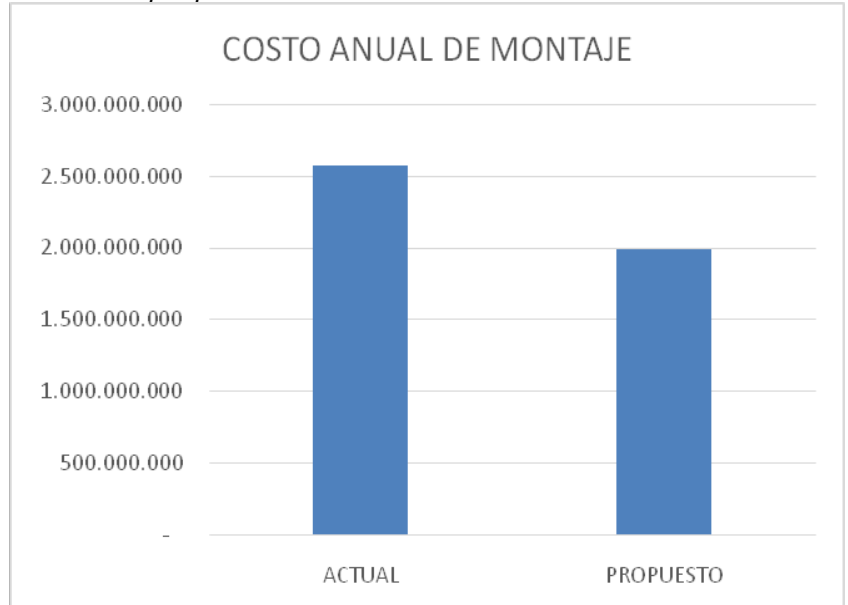

Figura 13: Análisis de costos del montaje con el método actual vs método propuesto.

Fuente: Elaboración propia.

Teniendo en consideración el método actual, se determinó que el método propuesto plasmado en un manual de procedimientos que busca guiar a la empresa analizada en la optimización de los procesos que implican el montaje de estaciones de radio base de manera eficiente y efectiva, es el más conveniente para la industria del caso de estudio, debido a que se logra disminuir los costos en el montaje de los mismos y disminuir la cantidad de multas, evitando pérdidas económicas importantes.

Tabla 7: Análisis de costos de multas del método actual vs método propuesto.

\begin{tabular}{|c|c|c|c|c|c|c|c|c|}
\hline \multirow{3}{*}{$\begin{array}{c}\text { Tipo de } \\
\text { sitio }\end{array}$} & \multicolumn{7}{|c|}{ Multas } & \multirow{3}{*}{$\%$ de ahorro } \\
\hline & \multicolumn{3}{|c|}{ Método actual } & \multicolumn{3}{|c|}{ Método propuesto } & \multirow{2}{*}{ Diferencia } & \\
\hline & $\mathrm{N}^{\circ}$ de multas & Costo unitario & Costo total & $\mathbf{N}^{0}$ de multas & Costo unitario & Costo total & & \\
\hline FS & 4 & 3.060 .000 & 12.240 .000 & 2 & 1.877 .149 & 3.754 .298 & 8.485 .702 & $69,33 \%$ \\
\hline LLM & 4 & 10.305 .000 & 41.220 .000 & 2 & 8.373 .825 & 16.747 .649 & 24.472 .351 & $59,37 \%$ \\
\hline COLLO & 8 & 2.718 .000 & 21.744 .000 & 4 & 2.153 .366 & 8.613 .464 & 13.130 .536 & $60,39 \%$ \\
\hline
\end{tabular}

Fuente: Elaboración propia. 


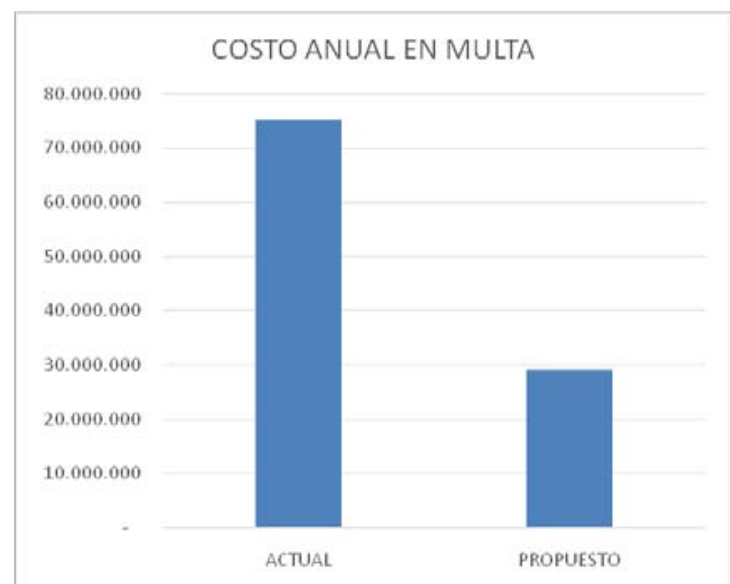

Figura 14: Análisis de costos de multas del método actual vs método propuesto. Fuente: Elaboración propia.

\section{Plan de ahorro}

El análisis económico realizado anteriormente, donde nos enfocamos en optimizar los procesos para ser más eficientes mediante el método propuesto, nos permitió establecer en la tabla 8 el plan de ahorro estimado:

Tabla 8: Plan de ahorro.

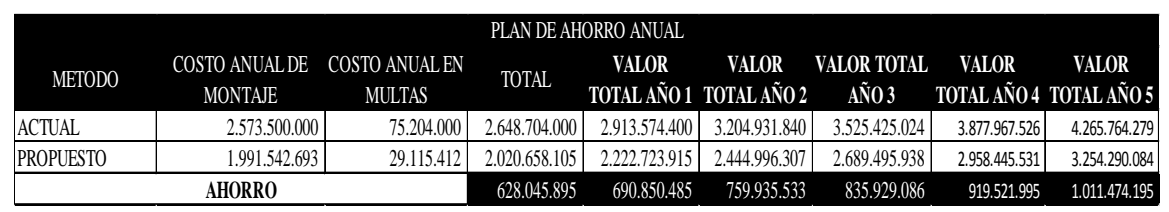

Fuente: Elaboración propia

\section{CONCLUSI ONES}

Este capítulo resume las conclusiones del trabajo final de grado, que provienen de los resultados obtenidos, ya expuestos en el capítulo anterior, y asegura el cumplimiento de los objetivos del proyecto.

El trabajo se realizó con la premisa de analizar el método de trabajo actual de la industria del caso de estudio. Con la finalidad de lograr identificar las falencias existentes en el método actual de la empresa se procedió a relevar información mediante entrevistas personales a los departamentos de Operaciones y de Logística, de manera a describir detalladamente las actividades de cada área que posee la empresa, así como los problemas detectados. También se revisó los antecedentes de penalizaciones (multas) por retraso en las obras solicitadas por los clientes de los últimos 5 años, ya que cabe destacar que el monto máximo de las multas se estableció que sea hasta el $9 \%$ (nueve por ciento) del costo del montaje, derivados principalmente por la falta de disponibilidad de materiales para el montaje de estaciones de radio base. 
Teniendo en consideración el análisis de la situación actual y apoyados sobre bases bibliográficas sobre estudios de métodos de trabajo, logística de materiales y monitoreo, se diseñó un método de trabajo optimizado acorde a las necesidades de la empresa, este método nos permite rentabilizar el proceso de montaje de una estación de radio base de cada tipo estudiado en el presente trabajo final de grado.

Como parte de la implementación del método propuesto, se establecieron el listado de materiales y de mano de obra de manera bien discriminada y detallada, con el objetivo de poder realizar un correcto seguimiento de los costos reales de la obra, considerando la cantidad de material exigido por cada tipo de estación de radio base.

Se diseñaron indicadores y un plan de monitoreo para asegurar la correcta aplicación y mantenimiento del sistema de trabajo propuesto. Esto facilita el control, al disponer de un flujo de trabajo estandarizado es posible realizar un control de las variables que figuran en cada indicador de los rubros identificados. Con esto se asegura tener datos cuantificables para la empresa que pueden ser traducidas de manera a dar una Retro alimentación para la mejora continua en los nuevos procesos diseñados para optimizar aún más los procesos.

Una vez diseñado el método de trabajo citado mencionado anteriormente, se procedió a la realización de un manual de implementación plasmando el nuevo método de trabajo. En el mismo se describe la distribución de tareas y responsabilidades de los departamentos, formularios de control de obra, formularios de costo de obra, paquetes de materiales, cronograma de montaje y flujograma de trabajo, en el cual debe volver a revisarse la documentación y realizar las mejoras con los datos obtenidos en la aplicación del método propuesto.

Se ha realizado el análisis de la viabilidad económica del nuevo método de trabajo propuesto y de sus posibilidades de implementación en la industria del caso de estudio, en donde a través del mismo se ha concluido que la implementación de este método mediante la utilización del manual propuesto se lograra optimizar los costos y aumentar la competitividad de la empresa, con la retroalimentación de los procesos, siendo fundamental un adecuado seguimiento de manera que a medida que se vayan montando las estaciones de radio base, se pueda lograr una mejora continua no solo en la eficiencia y en la reducción de costos sino en la competitividad en el mercado.

\section{REFERENCI AS BI BLI OGRAFI CAS}

Del Río Ruíz, E. (2018). Sistemas de telefonía fija y móvil. $1^{\circ}$ Edición. Madrid, España.: Ediciones Paraninfo S.A.

Hernández, R., Fernández, C., y Baptista, P. (2010) Metodología de la investigación. México. McGraw-Hill

Inzaurralde, M., Isi, J. \& Garderes, J. (2014). Telefonía Celular p.14. Montevideo: Facultad de Ingeniería - Universidad de la Republica de Montevideo.

León, S. (2015). Infraestructura para una BTS de telefonía móvil urbana. Sevilla: Depto. de Teoría de la Señal y Comunicaciones Escuela Técnica Superior de Ingeniería Universidad de Sevilla.

Torres, V. C. P. (2010). Calidad total en la atención al cliente. $1^{\circ}$ Edición. España Ideas propias Editorial SL.

Vivanco Vergara, M E. (2017). Los manuales de procedimientos como herramientas de control interno de una organización. Revista Universidad y Sociedad, 9(3), 247252. Recuperado en 19 de junio de 2020, de 
http://scielo.sld.cu/scielo.php?script=sci_arttext\&pid=S2218-

$36202017000300038 \& \operatorname{lng}=\mathrm{es} \&$ tlng=es.

Pérez Gutiérrez J.L. \& Lanza González E. B. (2014)."Manuales de procedimientos y el control interno: una necesaria interrelación," Observatorio de la Economía Latinoamericana, Servicios Académicos Intercontinentales SL, issue 201, August.Sitio web: https://ideas.repec.org/a/erv/observ/y2014i20118.html

Ramirez, R. (2017). Gestión de proyectos de instalaciones de telecomunicaciones. $1^{\circ}$ Edición. Madrid, España.: Ediciones Paraninfo S.A.

Cinco Días. (2017). La crónica detrás del primer teléfono celular en Paraguay. junio 30, 2019, de 5 Días. Sitio web: https://www.5dias.com.py/2017/09/la-cronicadetras-del-primer-telefono-celular-paraguay/

Comisión Europea. (2014). Glosario: Estación base (en telecomunicaciones). junio 27, 2019, de Comisión Europea Sitio web: http://ec.europa.eu/health/scientific_committees/opinions_layman/es/camposelectromagneticos/glosario/def/esTCÁionbase.html

Conatel. (2016). Plan Nacional de Telecomunicaciones Paraguay 2016-2020. junio 29, 2019, de Conatel Sitio web: https://www.conatel.gov. py/conatel/wpcontent/uploads/2019/10/rd.244.2016pnt-2016_2020.pdf 